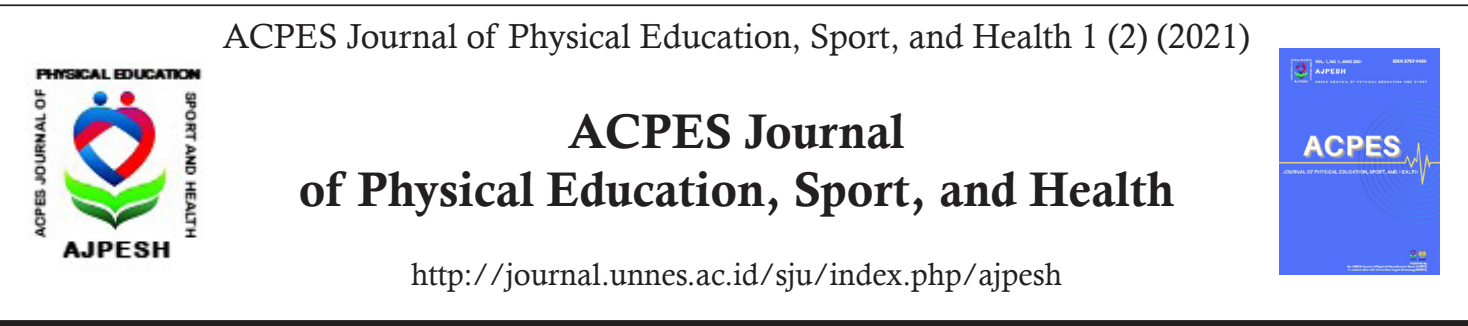

\title{
Validity of the 20 Meter Multi-Stage Shuttle Run Test (MSSRT) In Predicting VO2 Max
}

\author{
A. Shasi Kumar ${ }^{1,2 \bowtie}$ \\ ${ }^{1}$ Institute of Technical Education, Singapore \\ ${ }^{2}$ Edith Cowan University, Australia
}

DOI: https://doi.org/10.15294/ajpesh.v1i2.52587

\begin{tabular}{l} 
Article Info \\
\hline Article History \\
Submitted 2021-07-09 \\
Revised 2021-09-29 \\
Accepted 2021-11-17 \\
\hline Keywords \\
peer-to-peer scaffolding; \\
MYP Physical Education \\
\hline
\end{tabular}

\begin{abstract}
The purpose of this study was to investigate the validity of the 20 meter multi-stage shuttle run test (MST) in predicting VO2max compared to the directly measured reading from the laboratory. 4 male participants, who were diverse in terms of their ages and who were active physically, performed the MST and an incremental treadmill tests. Predicted VO2max values were obtained for the MST based on their stages and shuttles and actual VO2max values were obtained from the laboratory treadmill test. Results showed that all predicted $\mathrm{VO} 2$ max values for the MST over-predicted that of the actual VO2max values from the laboratory test. This study seems to suggest that there is no validity in the MST in predicting VO2max of an individual as compared to the actual $\mathrm{VO} 2 \mathrm{max}$ values from the laboratory.
\end{abstract}

\section{INTRODUCTION}

Aerobic fitness is the foundation by which most sports coaches and athletes build on before other elements like tactics and psychology are worked on. The maximal rate of oxygen consumption (VO2max) is considered the gold standard for measurement of this aerobic fitness (Sutton, 1992). The VO2max is an individual's threshold for producing energy aerobically and is often used in predicting aerobic endurance capacity (Boulay et al., 1985). There are a few ways to measure the VO2max. The direct measurement of oxygen uptake in the laboratory using the treadmill, cycling ergometer or rowing ergometer is one way. Due to the high cost and also availability of laboratory equipment, predictive measures of the VO2max were developed. One convenient method is the 20 meter multi-stage shuttle run test (MST) (Paradisis et al., 2014).

The MST has been widely used as a testing tool for fitness levels of individuals or teams. It is a field based continuous running test that requires participants to run between 2 lines, 20 meters apart, at an incremental pace dictated by an audio player (Lamb \& Rogers, 2007). Each stage of the MST is defined by a specific running velocity. Based on modifications made on the original MST by Leger \& Lambert (1982), the velocity will increase by $0.5 \mathrm{~km} / \mathrm{h}$ every stage. The MST will cease once participants fall out of the test. The predicted VO2max is then calculated based on the regression equation by Leger \& Lambert (1982) or simply, by cross-referencing the final stage and shuttle of the participants to a table of VO2max values (Brewer et al., 1988).

A number of studies have investigated the validity of the predicted $\mathrm{VO} 2 \mathrm{max}$ derived from the

$\square$ Correspondence Author:

10 Simei Avenue Singapore 486047

e-ISSN 2797-0426

E-mail: shasi_kumar_arumugam@ite.edu.sg 
MST. These studies compared VO2max values between MST and direct measurement in the laboratory. The VO2max values were under-predicted by an average of $9.3 \%$ for the MST compared to measurement in the laboratory (Berthoin et al., 1994). In another study by Saint-Gibson et al. (1998), on runners and squash players, the VO2max values for MST were under-predicted by an average of $6.8 \%$. These were consistent with findings from Sproule et al. (1993), who also concluded that the directly measured VO2max were higher compared to the values from MST. However, one study suggested that the predicted VO2max from the MST over-estimated the directly measured results (Flouris et al., 2005). This seems to suggest that the accuracy and validity of the MST in predicting VO2max is questionable. There were other studies though that concluded that the VO2max from MST is strongly related to results from the laboratory. The study by Brewer et al. (1988) found that the MST is a reliable source for estimates of the VO2max for active adult population. Given the fact that VO2max is an important factor in measuring an individual's aerobic fitness and the MST is frequently used as a testing method to derive the $\mathrm{VO} 2 \mathrm{max}$, it is of concern that there are varying results from past literature on the validity of the MST compared to the directly measured VO2max values.

Therefore the purpose of this study was to investigate the validity of the MST in predicting VO2max compared to the directly measured reading from the laboratory.

\section{METHODS}

\section{Participants}

4 participants volunteered to take part in the study. All 4 were males and were diverse in their ages with the oldest being 46 and the youngest being 26.3 of the participants were active in team sports like football and 1 participant is an endurance runner. All participants completed a medical questionnaire regarding their health status. All participants were cleared to undergo the tests. Prior to testing, the procedures of the tests were explained to the participants and all participants signed on written consents. The testing protocol was reviewed and approved by the Review Board and Ethical Committee of ECU.

\section{Experimental Procedures}

All 4 participants did a laboratory VO2max test followed by the MST. Both tests were done one day apart of each other. Participants completed the laboratory test on the Trackmaster Treadmill (TMX 30303C, Newton, USA). A nose clip and a sterilized mouthpiece, which was attached to a one way flow valve (Asahi AV Valve, Japan), were attached to the participants prior to the test. Participants started off at speed $6 \mathrm{~km} / \mathrm{hr}$ at a gradient 1.0 . The speed was increased by $1 \mathrm{~km} / \mathrm{hr}$ every minute with the gradient remaining constant throughout the test. All the participants were encouraged to give their best and till volitional exhaustion. The participants' resting heart rate was recorded before exercise and at the end of each minute of exercise using the Polar A3 heart rate monitor. The participants' rate of perceived exertion (RPE) from the Borg's scale readings were also taken after each minute of exercise. The test ended when participants cannot continue with the test due to exhaustion. The participants' RER, HR and VO2max were recorded. These readings were taken from the metabolic cart (Parvo, TrueOne 2400, USA). The participants were judged to have reached their VO2max when 3 of the following criteria as suggested by Davis (1995) were met: 1) levelling off of the VO2max (a change of $>2 \mathrm{ml} / \mathrm{kg} / \mathrm{min}$ over the last 60 seconds of the test), 2) RPE more than 17 using the Borg's scale, 3) RER $>1.05$, 4) Heart rate at termination $>95 \%$ of age predicted maximum HR and 5) volitional exhaustion.

The MST was conducted based on the original protocol by Leger \& Lambert (1982). It was conducted indoors on a carpeted surface. This provided traction so that slipping or sliding did not occur especially at the turning points. A 20 meter area was marked out using tapes and cones. There were adequate lanes for all 4 participants to go all at once. The test commenced with the participants running towards each $20 \mathrm{~m}$ end line in time for a "beep" sound from a audio player (20-m Shuttle Run Test CD, Australian Sports Commission). Running velocity increased by $0.5 \mathrm{~km} / \mathrm{hr}$ after every one minute stage. The test was stopped for any participant when they fail to reach the safe zone leading to the $20 \mathrm{~m}$ line for 2 consecutive occasions or when they fall out due to exhaustion. All participants were given encouragement throughout the test to give their best. Participants' RPE as well their HR was taken after the test. Their predicted VO2max based on their Level and Shuttle stage were then obtained from the chart from the Department of Sports Science, Loughborough University, 1988. 


\section{RESULT AND DISCUSION}

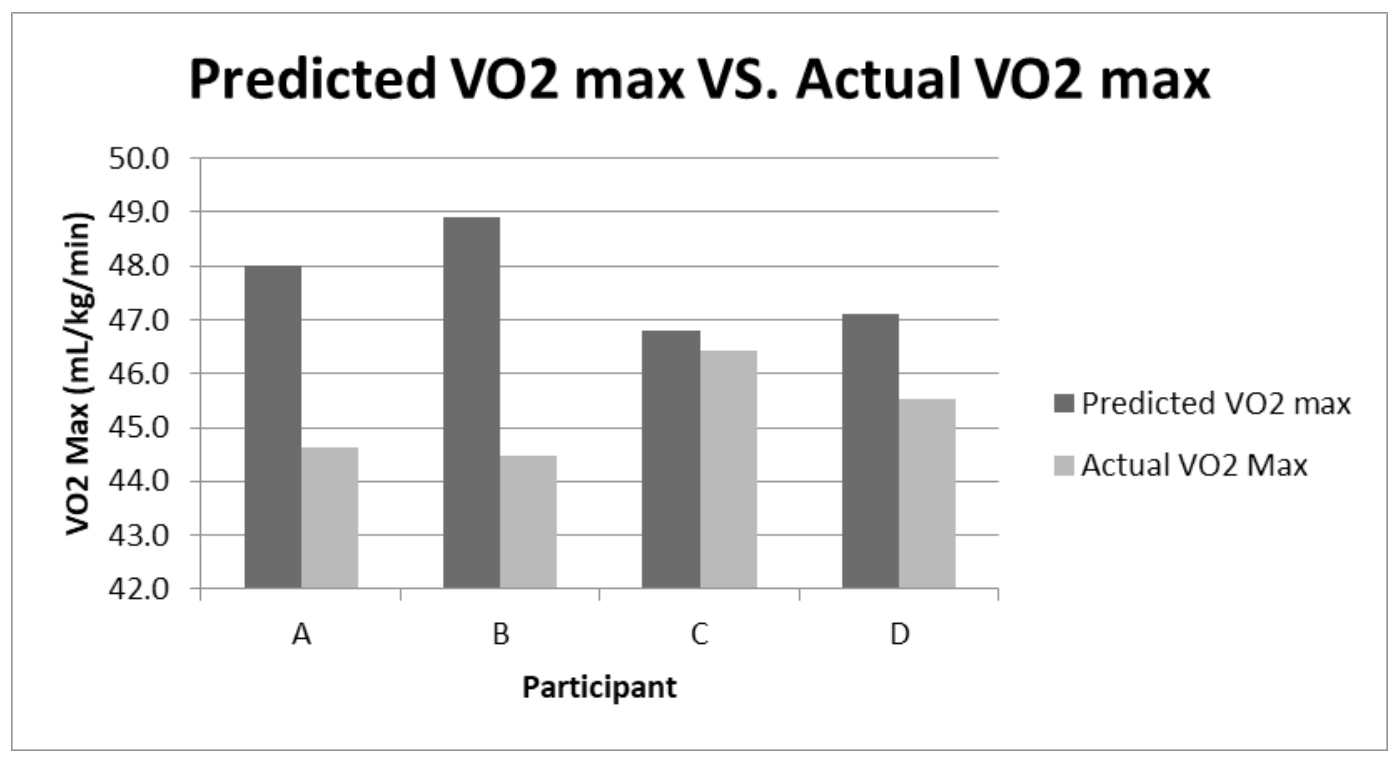

Figure 1. Difference between predicted VO2max (MST) and actual VO2max (laboratory)

Figure 1 shows the VO2max values from the MST (predicted) and the laboratory treadmill tests (actual). A's predicted VO2max is over-predicted at $48 \mathrm{ml} / \mathrm{kg} / \mathrm{min}$. His actual VO2max value is $44.64 \mathrm{ml} / \mathrm{kg} / \mathrm{min}$. B's predicted VO2max is also over-predicted by $4.42 \mathrm{ml} / \mathrm{kg} / \mathrm{min}$. His actual VO$2 \mathrm{max}$ value is $44.48 \mathrm{ml} / \mathrm{kg} / \mathrm{min}$. C's VO2max values, as can be seen in Figure 1 , shows a difference of $0.38 \mathrm{ml} / \mathrm{kg} / \mathrm{min}$. His actual VO2max is $46.42 \mathrm{ml} / \mathrm{kg} / \mathrm{min}$ compared to his predicted VO2max of $46.8 \mathrm{ml} / \mathrm{kg} / \mathrm{min}$. Finally, D's predicted $\mathrm{VO} 2 \mathrm{max}$ is also over-predicted at $47.1 \mathrm{ml} / \mathrm{kg} / \mathrm{min}$ compared to his actual VO2max reading of $45.52 \mathrm{ml} / \mathrm{kg} / \mathrm{min}$.

\begin{tabular}{lcccc}
\hline & \multicolumn{4}{c}{ Participants } \\
\hline & $\mathrm{n}=4)$ & & \\
\hline Variable & $\mathrm{A}$ & $\mathrm{B}$ & $\mathrm{C}$ & $\mathrm{D}$ \\
\hline & 44.6 & \multicolumn{2}{c}{46.4} \\
Actual V02max (ml/kg/min) & 44.5 & \multicolumn{2}{c}{45.5} \\
HR (bpm) & 199 & 186 & 181 & 170 \\
RER & 1.33 & 1.14 & 1.20 & 1.22 \\
RPE & 19 & 19 & 19 & 19 \\
\hline
\end{tabular}

Figure 2. Maximal exercise responses for the actual VO2max treadmill test

Figure shows the 4 of the maximal exercise responses for the laboratory treadmill test. Participant A's, B's, C's and D's HR hit 199, 186, 181 and $170 \mathrm{bpm}$ respectively at the end of the test. These values were more than $95 \%$ of their age-predicted maximum heart rate. The respiratory exchange ratio (RER) for all participants was above 1.05, with Khai having the highest ratio at 1.33 . Based on the Borg's scale for rate of perceived exertion (RPE), all the participants confirmed a value of 19 at the end of the treadmill test. As per Figure 1, the actual VO2max values show that Participant D has a value of $45.5 \mathrm{ml} / \mathrm{kg} / \mathrm{min}$, which was the highest amongst the 4 participants.

The purpose of this study was to investigate the validity of the MST in predicting VO2max compared to the directly measured reading from the laboratory. The study was done as the MST is one of the most common tests administered on teams or individuals to find their VO2max levels. Therefore it is important to find out the validity of the MST as a predictor of VO2max compared to the actual VO2max values from a laboratory treadmill test.

Figure 1 shows the VO2max values from the MST (predicted) and the laboratory treadmill tests (actu- 
al). The predicted VO2max values from the MST for all the participants in this study over-predicted the actual VO2max values from the treadmill test. Only Participant $\mathrm{C}$ had VO2max level which was very close, with the difference being only $0.38 \mathrm{ml} / \mathrm{kg} / \mathrm{min}$. These findings were not consistent with studies by Berthoin et al., (1994) and Gibson et al., (1998), who concluded that the predicted VO2max values from the MST under-predicted the values from the treadmill test. However, the present study seems to suggest the relationship between an endurance based athlete or individual and an individual who is part of a team sport to the VO2max values achieved, as suggested in a study by Gracin et al. (2003). It was found that endurance based athletes or individuals had a lower VO2max value from the MST than individuals from team sports background. This was believed to be caused by the fact that the physiological and metabolic responses are different in endurance athletes and team sports athletes (Hamilton et al., 1991). The participants in the present study consists of 3 individuals who are part of team sports and 1 (Participant C), who is an endurance athlete. Therefore, this study is consistent with that of Gracin et al., (2003). Participant C, with the closest relation between VO2max values for the MST and the treadmill test, is an endurance runner. Therefore his VO2max for the MST was lower than that of the other 3 participants, This study suggests that the reason for the significant over-prediction for the MST for the other 3 participants could be due to the fact that they are accustomed to the stop starts or intermittent nature of their sport and therefore scoring higher VO2max values than Participant $\mathrm{C}$, who is more accustomed to a more continuous type of exercise.

Another reason for the difference in the $\mathrm{VO} 2 \mathrm{max}$ values for both tests could be due to the fact that absolute values were used for the MST instead of values relative to the participants' body mass. Gore (2000) opined that this method of utilising absolute values puts the validity of the test under scrutiny. The present study agrees as participants with different body masses will physiologically adapt differently to the tests demands.

Another limitation and also a reason for the varying results between the 2 tests could be due to the sample size of 4 males. The size is not representative of the population and the homogeneity of the sample (all males with sporting backgrounds) can be biased as all of them could have taken the MST before and were familiar with the testing protocols and strategy to hit a higher stage and shuttles in the test.

Astrand et al. (2003) suggested that participants feeling restricted in the laboratory test could hinder their performance. This present study agrees as the mouthpiece with the one-way flow valve could be uncomfortable and unrealistic as well. This could be a reason for the under-estimation of the VO2max from the treadmill test.

\section{CONCLUSION}

Results from this present study suggests that validity of the MST for measuring VO2max is questionable. The VO2max values from the MST were over-predicted as compared to the actual VO2 max values from treadmill test. Possible reasons and limitations for this present study were taken into account for this over-prediction and highlighted in the discussion section. Therefore, future direction for validity testing of the MST in measuring VO2max should factor in those pertinent points in order to make an accurate judgement of the subject.

\section{ACKNOWLEDGEMENT}

Much appreciation to Edith Cowan University, Australia with special mention to the Physical Education departments of the Institute of Technical Education, Singapore.

\section{REFERENCES}

Astrand, P., Rodahl, K., Dahl, H. \& Stromme, S. 2003. Textbook of Work physiology: Physiological basis of Exercise. United State: Human Kinetics.

Boulay, M., Lortie, G., Simoneau, P., Hamel, C., Leblanc, C. \& Bouchard, C. 1985. Specificity of Aerobic and Anaerobic Work Capacities and Power. International Journal of Sports Medicine, 6 (6): 325-328. https://doi.org/10.1055/s-2008-1025864

Brewer, J., Ramsbottom, R. \& Williams, C. 1988. A Progressive Shuttle Run Test to Estimate Maximal Oxygen Uptake. British Journal of Sports Medicine, 22 (4): 141-144. http://dx.doi.org/10.1136/ bjsm.22.4.141

Berthoin, S., Gerbeaux, M., Thurpin, E., Guerrin, F., Lensel-Corbeil, G. \& Vendendorpe, F. 1994. Comparison of Two Field Tests to Estimate Maximum Aerobic Speed. Journal of Sports Science, 12 (4): 355-362. https://doi.org/10.1080/02640419408732181 
Davies, J. 1995. Physiological Assessment of Human Fitness (p. 14). Champaign, IL: Human Kinetics.

Flouris, A., Metsios, G. \& Koutedakis, Y. 2005. Enhancing the Efficacy of the $20 \mathrm{~m}$ Multistage Shuttle Run Test. British Journal of Sports Medicine, 39 (3): 166-170. http://dx.doi.org/10.1136/ bjsm.2004.012500

Gracin, M., Mille-Hamard, L., Devillers, S., Delattre, E. \& Dufour, S. 2003. Influence of the Type of Training Sport Practiced on Psychological and Physiological Parameters during Exhausting Endurance Exercises. Journal of Perceptual Motor Skills, 97: 1150-1162. http://dx.doi.org/10.2466/ PMS.97.8.1150-1162

Gore, C. 2000. Physiological tests for elite athletes. Champaign,, IL: Human Kinetics.

Hamilton, A., Nevill, M., Brooks, S. \& Williams, C. 1991. Physiological Responses to Maximal Intermittent Exercise. Journal of Sports Science, 9 (4): 371-382. https://doi. org/10.1080/02640419108729897

Lamb, K. \& Rogers, L. 2007. A Re-appraisal of the Reliability of the $20 \mathrm{~m}$ Multi-stage Shuttle Run Test. European Journal of Applied Physiology, 100 (3): 287-292. http://dx.doi.org/10.1007/ s00421-007-0432-9

Leger, L. \& Lambert, J. 1982. A Maximal Multi Stage 20 meter Shuttle Run Test to Predict VO2max. European Journal of Applied Physiology, 49 (1): 1-12. https://doi.org/10.1007/bf00428958

Paradisis, G., Zacharogiannis, E., Mandila, D. \& Smirniotoy, A. 2014. Multi-Stage 20-m Shuttle Run Fitness Test, Maximal Oxygen Uptake and Velocity at Maximal Oxygen Uptake. Journal of Human Kinetics, 41 (1): 81-87. http://dx.doi.org/10.2478/hukin-2014-0035

Saint-Gibson, A., Broomhead, S., Lambert, M. \& Hawley, J. 1998. Prediction of Maximal Oxygen uptake from a 20-meter shuttle run as measured Directly in Runners and Squash Players. Journal of Sports Science, 16 (4): 331-335. https://doi.org/10.1080/02640419808559361

Sproule, J., Kunalan, C., McNeill, M. \& Wright, H. 1993. Validity of 20-MST for Predicting VO2max of Adult Singaporean Athletes. British Journal of Sports Medicine, 27 (3): 202-204. https:// dx.doi.org/10.1136\%2Fbjsm.27.3.202

Sutton, J. 1992. Limitations to Maximal Oxygen Uptake. Journal of Sports Medicine, 13, 127-133. https://link.springer.com/article/10.2165/00007256-199213020-00008 